\title{
Alcohol Effects on TNF- $\alpha$ and IL-10 Production in an Ex-Vivo Model of Whole Blood Stimulated by LPS
}

Alexandra Gavala ${ }^{1 *}$, Pavlos Myrianthefs ${ }^{1}$, Kyriaki Venetsanou ${ }^{1}$, George Baltopoulos ${ }^{1}$ and Georgios Alevizopoulos ${ }^{2}$

${ }^{1}$ Faculty of Nursing, University of Athens, ICU at "Agioi Anargyroi" General Hospital, Greece

${ }^{2}$ Department of Psychiatry and Behavioral Sciences, "Agioi Anargyroi" General Hospital, Greece

\begin{abstract}
Alcohol exposure is related to increased susceptibility to infections. We investigated the effect of acute exposure to alcohol on pro- and anti-inflammatory cytokine production in an ex-vivo model of whole blood stimulation with lipopolysacharide (LPS). Ten $\mathrm{ml}$ of whole blood were taken from 24 healthy volunteers (all men) aged $36.5 \pm 1.4$ years. Each sample was transferred into two tubes, without and with EDTA as anticoagulant, under sterile conditions. We had 14 groups: the control group in which whole blood was incubated without any other intervention, the LPS group in which whole blood was stimulated with LPS alone, and 12 alcohol groups with (6 groups) and without LPS stimulation (6 groups) using six different doses of alcohol (5, 12.5, 25, 50, 100 and 200mM). LPS (500pg) was added after pretreatment with alcohol for 10 minutes. Blood samples were diluted 1:10 in RPMI 1640 culture medium

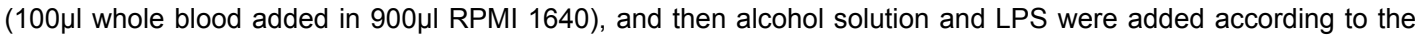
study protocol for incubation period of 4 hours at $37^{\circ} \mathrm{C}$. Cell culture supernatants were isolated with centrifugation at $1,800 \mathrm{rpm}$, for $5 \mathrm{~min}$ at room temperature and stored at $-70^{\circ} \mathrm{C}$ until measurements. Cytokine levels were determined in culture supernatant with the ELISA method. Alcohol had no effect on cytokine production when incubated with whole blood alone. TNF- $\alpha$ IL-6 and IL-10 significantly increased after LPS stimulation. Alcohol had no effect on IL-6 production after LPS challenge but significantly decreased TNF- $\alpha$ and IL-10 production in the presence of LPS challenge at $25 \mathrm{mM}$ to $200 \mathrm{mM}$ in a dose dependent manner. Conclusively TNF- $\alpha$ and IL-10 were significantly decreased after alcohol exposure in a dose depended manner in a model of whole blood stimulation with LPS exvivo expressing both suppression of pro- and anti-inflammatory response.
\end{abstract}

Keywords: Alcohol; Cytokines; Lipopolysacharide; TNF- $\alpha$; IL-6; IL-10

Abbreviations: LPS $=$ lipopolysaccharide; TNF- $\alpha=$ Tumor necrosis factor-alpha; IL-6 = Interleukin-6; IL-10 = Interleukin-10

\section{Introduction}

Alcohol (ethanol) abuse is a global problem affecting human health in many ways. These effects are complex and related to many factors (i.e. quantity of alcohol taken, duration and pattern of drinking, gender, genetic factors).

Alcohol consumption can cause major health problems, including liver cirrhosis and other ailments. Prolonged and high alcohol consumption can lead to the development of alcoholic liver disease amongst the most serious liver diseases. Although mild alcoholic hepatitis can be asymptomatic, the disease may develop to a more serious state due to liver hepatocyte damage, leading to an increased death risk up to $20 \%$. If heavy alcohol intake continues alcoholic hepatitis persists and progresses to liver cirrhosis (The International Liver Congress 2012 EASL).

Both acute and chronic alcohol use can affect the innate and acquired immune responses, impairing antimicrobial defense against bacteria and viruses [1-5]. Chronic alcohol abuse has been associated with immunosuppression, increased incidence of infections (especially bacterial pneumonia) and increased morbidity and mortality [1-5]. Alcohol abuse is associated with increased incidence of several serious infections including pneumonias, tuberculosis, chronic hepatitis $\mathrm{C}$ infection and increased susceptibility to HIV infection [4,6-10]. Additionally, acute alcohol exposure increases the risk of infection in trauma and burn patients [11-14].

Alcohol abuse significantly affects structural and cell-mediated host defense mechanisms. Extensive evidence suggests that alcohol ingestion (acute or chronic) impairs the integrity of the physical barriers of GI and respiratory tracts [1].

Alcohol overexposure modifies intestinal permeability which may cause translocation of bacterial-derived molecules, such as lipopololysaccharides-LPS Gram negative bacteria component), from the intestinal lumen to the portal vein (into the bloodstream supplying the liver). A variety of cells are activated by these molecules in the liver (epithelial cells, Kuppfer cells, hepatic cells) causing inflammation and organ injury [1].

LPS (bacterial endotoxin) is the most potent stimulus for TNF-a production and activator of cytokine cascade. Increased LPS levels in the blood form a complex with the LPS-binding protein that in turn binds to the CD14 receptor on the macrophage surface, inducing their stimulation and therefore the production of pro-inflammatory cytokines, especially Tumor Necrosis Factor alpha (TNF- $\alpha$ ).

Several studies in animals and humans show that alcohol exerts an immunosuppressive effect in a variety of immune cells including polymorphonuclear cells, monocytes/macrophages and Tlymphocytes

*Corresponding author: Alexandra Gavala, Faculty of Nursing, University of Athens, ICU at "Agioi Anargyroi" General Hospital, Greece, Tel: +30-210-3501195; Fax: +30-210-6231949; E-mail: alexandra.gavala@gmail.com

Received September 25, 2015; Accepted October 27, 2015; Published November 03, 2015

Citation: Gavala A, Myrianthefs P, Venetsanou K, Baltopoulos G, Alevizopoulos $G$ (2015) Alcohol Effects on TNF- $\alpha$ and IL-10 Production in an Ex-Vivo Model of Whole Blood Stimulated by LPS. J Psychiatry 18: 339 doi:10.4172/2378-5756.1000339

Copyright: @ 2015 Gavala A, et al. This is an open-access article distributed under the terms of the Creative Commons Attribution License, which permits unrestricted use, distribution, and reproduction in any medium, provided the original author and source are credited 
by suppressing chemotaxis, inflammatory response and inflammatory mediators including cytokines $[3,6,11,15-19]$.

It is believed that impaired inflammatory cytokine production after acute alcohol exposure is associated with impaired host defense $[5,20]$. Most research studies in humans hitherto examined alcohol effects in cell cultures incubated with alcohol or cell cultures received from blood donors after alcohol consumption. There are limited data regarding ex-vivo human whole blood studies examining the effect of alcohol on cytokine production in the presence of lipopolysaccharides (LPS) stimulation.

The purpose of the study was to investigate the effect of acute alcohol exposure using different doses of alcohol on cytokine production in a model of ex-vivo whole blood stimulation with LPS.

\section{Materials and Methods}

\section{Subjects and Protocol}

Peripheral blood was obtained from 24 healthy male volunteers and immediately transferred for laboratory processing according to the study protocol as previously described by both others and ours [21-23].

Before taking blood, a complete medical history and clinical examination (smoking, drugs, diseases etc) was performed and informed consent was obtained by all participants. This ensured the healthy status of the volunteers as well as permission to obtain blood for these experiments. Ethical approval was obtained from Hospital Scientific Council and Ethics Committee and the Athens University School of Nursing Ethics Committee as well.

Totally, ten (10) $\mathrm{ml}$ of whole blood sample was collected from each individual and transferred into two (2) vacutainer tubes under sterile conditions as follows: five (5) $\mathrm{ml}$ in one tube without anticoagulant for serum (baseline) cytokine measurements before any intervention and five (5) $\mathrm{ml}$ with EDTA as anticoagulant. All blood samples were then processed accordingly.

The blood samples were centrifuged at $2.000 \mathrm{rpm}$, for $10 \mathrm{~min}$ at room temperature to isolate the serum and stored for baseline measurements of cytokine levels.

A number of 14 different experimental groups were developed as follows: The control group in which whole blood was incubated with no other intervention. The LPS group in which, whole blood was stimulated with LPS alone. The alcohol groups with 6 different alcohol dose each $(5,12.5,25,50,100$, and $200 \mathrm{mM})$. Each dose group was divided into two series the first with and the second without LPS stimulation. The LPS was added 10 minutes after the alcohol pretreatment. The groups resulted to a total of 140 experimental runs.

All samples were incubated for 4 hours at $37^{\circ} \mathrm{C}$, in humidified $\mathrm{CO}_{2}$ incubator. For the experiment we used $0.1 \mathrm{ml}$ of whole blood, $0.9 \mathrm{ml}$ RPMI, $500 \mathrm{pg} / \mathrm{ml}$ of LPS final concentration, and alcohol at 5, 12.5, 25, 50,100 and $200 \mathrm{mM}$ as mentioned previously.

Briefly, $0.1 \mathrm{ml}$ whole blood was diluted 1:10 in $0.9 \mathrm{ml}$ RPMI 1640 culture medium, and then alcohol solution and LPS were added according to the study protocol for incubation period of $4 \mathrm{~h}$.

Cell culture supernatants were isolated with centrifugation at 1,800 $\mathrm{rpm}$, for $5 \mathrm{~min}$ at room temperature and stored at $-70^{\circ} \mathrm{C}$ until cytokine measurements.

\section{Reagents and equipments}

Absolute ethylic alcohol (EtOH) was purchased from Merck,
Germany. LPS from E. coli (0111:B4) was purchased from Sigma Chemical Co (St Luis, MO, USA). Culture medium RPMI 1640 was purchased from Biochrom, Gmbh, Germany. Tumor Necrosis Factor- $\alpha$ (TNF- $\alpha$ ), Interleukin-6 (IL-6), and Interleukin-10 (IL10) were determined using commercially available human specific enzyme-linked immunoassays kits (ELISA kit, purchased from INVITROGEN, Camarillo-USA) according to the manufacturer's instructions. The absorbance of final coloured products was measured as optical density (OD) with microplate reader (Sunrise Absorbance Reader, Salzburg, Austria GMBH), at dual wavelength 450/630 nm. Cytokines concentrations were calculated with a standard curve using 4-parameters algorithm. All kits for cytokines are human specific with sensitivity of detecting levels for TNF- $\alpha>1.7 \mathrm{pg} / \mathrm{ml}$, IL- $6>2 \mathrm{pg} / \mathrm{ml}$, and IL- $10>1 \mathrm{pg} / \mathrm{ml}$.

\section{Measurements}

Pro- inflammatory cytokines studied included TNF- $\alpha$, IL-6, and anti- inflammatory included IL-10. At baseline White Blood Cells (WBC) and differential, serum TNF- $\alpha$, IL-6, IL-10, performed. At $4 \mathrm{~h}$ we measured TNF- $\alpha$, IL- 6 , and IL-10 in the cell culture supernatants.

\section{Statistics}

Values are expressed in mean \pm SEM. Statistical analysis was performed using One-way analysis of variance ANOVA Tukey's Multiple Comparison Test suitable for multiple comparisons. Statistical significance was determined at the level of $\mathrm{p}<0.05$.

\section{Results}

We included 24 men mean aged $36.5 \pm 1.4$ years. Serum baseline cytokines were for TNF- $\alpha 2.3 \pm 1.2$; while IL- 6 and IL-10 levels were non-detectable. White blood cells (WBC) were $5758 \pm 239.5 / \mathrm{mm}^{3}$ and differentials were polymorphonuclear $3363 \pm 191.8 / \mathrm{mm} 3$, lymphocytes $1958 \pm 103.1 / \mathrm{mm}^{3}$, and monocytes $374.4 \pm 30.9 / \mathrm{mm}^{3}$.

Cytokine production after LPS stimulation and alcohol exposure is shown in Figure 1. TNF- $\alpha$, IL-6 and IL-10 significantly increased in the supernatants after LPS stimulation. Alcohol significantly decreased TNF- $\alpha$ production in the presence of LPS challenge at concentrations between $25 \mathrm{mM}$ and $200 \mathrm{mM}$. This effect was most profound at doses of 100 and $200 \mathrm{nM}$. Alcohol also significantly decreased IL-10 production in the presence of LPS challenge at $25 \mathrm{mM}$ to $200 \mathrm{mM}$ in a dose dependent manner.

No discernible effect was detected when Ethylic alcohol (ETH) incubated in different increased doses $(5,12.5,25,50,100$ and $200 \mathrm{mM}$, not shown in Figure 1) with whole blood alone

\section{Discussion}

In this study we found that levels of both TNF- $\alpha$ and IL-10 were significantly decreased after alcohol exposure in a dose dependent manner in a model of whole blood stimulation with LPS ex-vivo. In contrast, alcohol had no effect on IL-6 production. Alcohol did not affect all three cytokine production when added alone to whole blood indicating that, in the presence of LPS challenge, TNF- $\alpha$ and IL10 production was affected through common molecular pathways. However, this observation needs further investigation.

Several in vitro and in vivo studies showed that acute exposure to alcohol decreased proinflammatory cytokines production after stimulation with a variety of stimuli, including both gram-negative and gram-positive bacterial products. Literature suggests that TNF- $\alpha$ is 


\section{TNF-a}

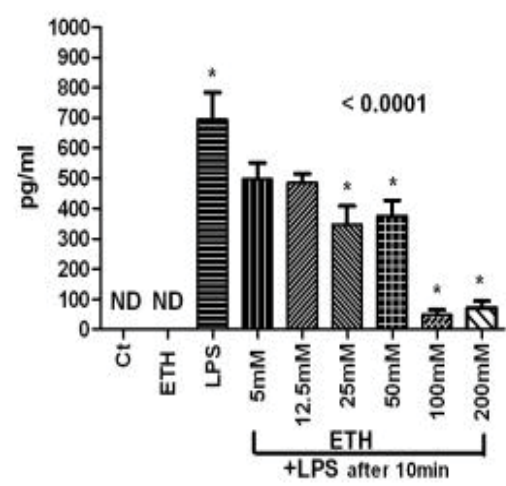

IL-6

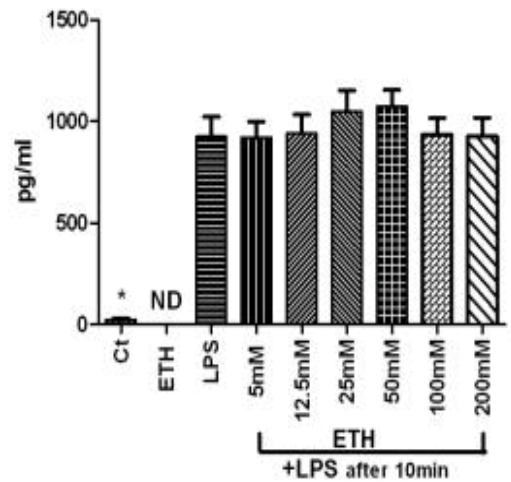

IL-10

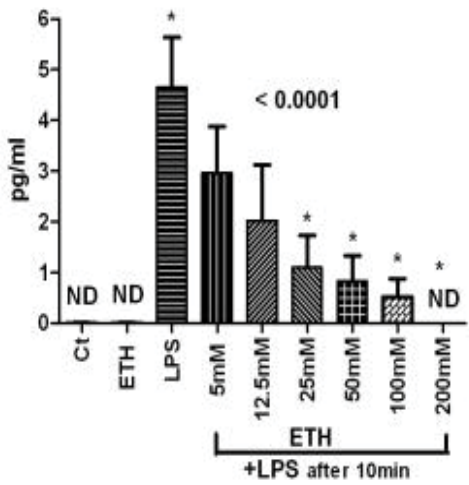

Figure 1:Cytokine production of whole blood ex-vivo in different groups studied.

$\mathrm{Ct}=$ Control, whole blood with culture medium alone; $\mathrm{ETH}=$ whole blood with six different doses of ethylic alcohol (Ethanol 5, 21.5, 25, 50, 100, 200mM, not shown); LPS = whole blood with 500 pg of LPS; ND= non detectable.

decreased after alcohol exposure in various different models. The latter is a consistent finding among different species (human and animal), different types of cells (peripheral blood monocytes, dendritic cells, and macrophages), and different routes of alcohol administration (in vivo or in vitro).

It was found that acute exposure to alcohol induced a dosedependent decrease in TNF- $\alpha$ production by monocytes which occurs at multiple levels since alcohol acts both at cellular membrane and at the intracellular level [11,24-26].

According to literature acute alcohol exposure inhibits TLR4 mediated signaling and subsequent NF- $\mathrm{kB}$ activation and cytokine production [24,26]. Acute alcohol exposure results in a significant post-transcriptional and post-translational suppression of TNF-a production [24].

It has also been reported that acute ethanol exposure not only decreased the activation of TACE but also the release of TNF- $\alpha$. Furthermore, data supports that decreased TNF- $\alpha$ production involves decreased transcription mechanisms [11,26].

According to evidence it is suggested that both TACE and TLR4 are possible targets of immunosuppressive effects of ethanol [26].

Nelson et al. in an in vivo rat experimental study found that acute alcohol intoxication significantly decreased TNF- $\alpha$ production in serum and bronchoalveolar lavage fluid after LPS stimulation [27]. Acute ethanol exposure decreased multiple TLR agonists, induced IL-6 and TNF- $\alpha$ production by murine macrophages [28], and suppressed polyinosinicpolycytidylic acid induced IL-6 production in murine peritoneal macrophages [29].

Experimental studies with isolated human monocytes showed that acute alcohol exposure reduced the production of pro-inflammatory cytokines after provocation with various bacterial products.

In human monocytes alcohol exposure decreased TNF- $\alpha$, IL$1 \beta$ and IL- 6 production after LPS or staphylococcal enterotoxin A and B stimulation [30-33]. Verma et al. found that alcohol (acute administration) reduced LPS induced TNF-a production by human peripheral blood monocytes, and that this reduction was dose dependent and statistically significant in the 25-150 mM, alcohol concentration range [30].
Szabo et al. showed that in vitro acute alcohol exposure $(\mathrm{EtOH}$ $25 \mathrm{mM}, 100 \mathrm{mM}$ ) significantly inhibited the production of TNF- $\alpha$, IL-1-beta, and IL-6 when induced by superantigen (Staphylococcus enterotoxins B and A) in human monocytes [31]. The same authors subsequently showed that in vitro acute alcohol treatment suppressed human blood monocytes production of TNF- $\alpha$ and IL- $1 \beta$ after provocation with LPS and staphylococcal enterotoxin B [32].

More recent studies have shown similar results. Pang et al. showed that in vitro co-administration of alcohol, when processed as an acute alcohol challenge ( $25 \mathrm{mM}$ ethanol) with TLR4 ligand $(0.1 \mathrm{ug} / \mathrm{ml} \mathrm{LPS})$ resulted in a significant inhibition of TNF- $\alpha$ production by human monocytes (at 6 hours) [25].

Nair et al., which is the only study examining the effect of alcohol on whole blood and total mononuclear cells TNF- $\alpha$ production in healthy volunteers ex-vivo, found a significant decrease in TNF- a production after LPS stimulation at a dose of $25 \mathrm{mM}$ which is in accordance with our findings.

However, in this study, E. coli (L-2630E) derived LPS were used in a significantly higher concentration of $10 \mu \mathrm{g} / \mathrm{ml}$ as compared to our study $(500 \mathrm{pg} / \mathrm{ml})$ [34].

Additionally, in vitro and in vivo studies showed that acute and chronic alcohol exposure enhanced the production of antiinflammatory cytokines $[25,35]$.

Acute alcohol exposure in vivo and in vitro induced IL-10 production in human monocytes, with or without LPS stimulation [32,36-40,41], and thus may interfere with the cell-mediated and humoral immunity by both reducing inflammatory cytokine production and preventing T-cell proliferation $[17,36,42]$.

Szabo et al. found that in vitro acute alcohol treatment induced the production of transforming growth factor-beta and IL-10 by human blood monocytes [32]. Mandrekar et al. found that both low $(25 \mathrm{mM})$ and high $(100 \mathrm{mM})$ alcohol concentrations significantly increased IL-10 production in LPS stimulated isolated peripheral blood human monocytes in vitro after $18 \mathrm{~h}$ culture. In contrast, although the combined stimulation of $25 \mathrm{mM}$ ethylic alcohol and LPS did not significantly changed IL-10 production after $10 \mathrm{~h}$ stimulation, $100 \mathrm{mM}$ alcohol and LPS significantly decreased monocyte IL-10 production 
than LPS alone [36]. Several other studies using a completely different protocol including drinking of alcohol in healthy volunteers and blood receipt after 18-24 hours, which was stimulated with or without LPS, found a significant increase in IL-10 production [37-40]. These findings do not contrast our results since a shorter timeframe was applied in our experiments in addition to the use of whole blood in an ex-vivo environment. According to our data alcohol significantly decreased IL-10 production in the presence of LPS challenge at $25 \mathrm{mM}$ to 200 $\mathrm{mM}$. In a previous study in healthy volunteers, Szabo found a transient decrease in IL-10 production in the in vivo ethanol-exposed whole blood samples (obtained $4 \mathrm{hr}$ after alcohol consumption) in response to ex vivo stimulation with LPS [43].

Such discrepancies may be related to the methodological approach (i.e., alcohol administration in vivo vs. ex vivo vs. in vitro exposure), timing of LPS challenge (before or after exposure to alcohol which was administered in vivo or direct to cell cultures), type of samples used to evaluate the cytokine production by alcohol administration (i.e., human blood monocyte cultures vs. human whole blood), and duration of exposure to alcohol (i.e., 4hours vs. 18-24hours) [44]. In addition, Pruett et al. suggested that research regarding the effect of alcohol on IL-10 production conducted in vitro should be interpreted with caution and confirmed in vivo $[45,46]$.

In our study, we investigated the effect of acute exposure of alcohol on IL-10 whole blood production 4 hours after LPS stimulation. Anti -inflammatory IL-10 is known to peaks after 6 hours of LPS challenge. Thus in order to have clearer image further investigation is needed.

We used an experimental model of cytokine production based on ex vivo whole blood stimulation, which has significant advantages compared to stimulation of isolated monocytes from peripheral blood samples. The advantages of ex-vivo whole blood model as compared to in vitro studies are summarized elsewhere. In summary, whole blood culture assays retain all blood components and maintain normal operating environment for cell to cell interactions and there are also present circulating stimulatory and inhibitory mediators necessary for cell viability. Also, this method has been suggested to be most appropriate to study cytokine production in an environment that reproduces physiological phenomena, is very simple and quicker, less expensive and can be readily adapted to use in field studies [21,47].

We used a wide range of alcohol concentrations varying from 5 to $200 \mathrm{mM}$ (milimoles/liter-mmol/L). This choice was based on observations suggesting that $5-150 \mathrm{mM}$ concentrations are comparable to physiologically occurring blood alcohol levels after mild, moderate and heavy-binge alcohol intake. Specifically, alcohol concentrations of $25 \mathrm{mmol} / \mathrm{L}$ used in vitro approximate blood alcohol levels of $0.1 \%$ achieved after a single-dose moderate drink. Also, concentrations of $100 \mathrm{mmol} / \mathrm{L}$ correspond to blood alcohol levels of approximately $0.4 \%$, representing the highest levels typically observed in acute alcohol consumption by alcoholics or incidentally by binge drinkers [48]. The concentration of $200 \mathrm{mM}$ alcohol is not relevant to humans and was selected as an upper limit of alcohol intoxication and its effects on cytokine production.

Our study has several limitations. Firstly, our study is descriptive and not mechanistic. Secondly, while whole blood culture is a sensitive method and thus able to determine the ex-vivo pro-inflammatory cytokine production in response to endotoxin stimuli, is still limited compared to in vivo conditions. This is because such an ex-vivo model lacks both the complex physiological effects of alcohol and the systemic alcohol metabolism. Additionally, despite whole blood culture assays have significant advantages compared to ex vivo stimulation of isolated cells, there is a number of unpredictable and unknown factors, having confounding effect on the possible results. Whole blood models explore a wide cell population in which the variety of cells is unknown. Although cytokine production, as measured in both whole blood and mononuclear cell cultures, is significantly correlated, changes in the number of viable cells - producing cytokines or changes in the ability of these cells to produce cytokines - could result in variations in cytokine production under either different circumstances or between different individuals leading to misinterpretation of findings [21,47].

\section{Conclusion}

Alcohol per se did not affect cytokine production when incubated with whole blood samples. Alcohol decreased TNF- $\alpha$ and IL-10 production in a dose depended manner from whole blood stimulated with LPS ex-vivo. Our observations indicate a suppressive effect of acute alcohol exposure on both pro- and anti-inflammatory pathways. These data suggest that acute alcohol intoxication has an immunomodulatory activity in the presence of infection (LPS challenge), depending on the alcohol concentration. Despite the suppressive effect on proinflammatory response, alcohol moderate intoxication $(25-50 \mathrm{mM})$ causes also greater significant suppression of the anti-inflammatory response (figure 1). This fact may indicate a stronger early proinflammatory response in humans, in the presence of LPS challenge, predisposing more severe clinical manifestations of infection.

Further research is needed for the mechanism, underlying the effect of acute alcohol on the expression of cytokines in the manifestation of infection, to be clarified.

\section{Acknowledgements}

We are thankful to Prof. C. Spiliopoulou, Prof. S. Athanaselis and Prof. G. Filntisis for reviewing and commenting on the manuscript.

\section{Conflict of Interes}

There is no conflict of interest by any of the authors

\section{References}

1. Molina PE, Happel KI, Zhang P, Kolls JK, Nelson S (2010) Focus on: Alcohol and the immune system. Alcohol Res Health 33: 97-108.

2. Szabo G, Mandrekar P (2009) A recent perspective on alcohol, immunity, and host defense. Alcohol Clin Exp Res 33: 220-232.

3. Goral J, Karavitis J, Kovacs EJ (2008) Exposure-dependent effects of ethanol on the innate immune system. Alcohol 42: 237-247.

4. Nelson S, Kolls JK (2002) Alcohol, host defence and society. Nat Rev Immunol 2: 205-209.

5. Cook RT (1998) Alcohol abuse, alcoholism, and damage to the immune system--a review. Alcohol Clin Exp Res 22: 1927-1942.

6. Happel KI, Nelson S (2005) Alcohol, immunosuppression, and the lung. Proc Am Thorac Soc 2: 428-432.

7. Zhang P, Bagby GJ, Happel KI, Raasch CE, Nelson S (2008) Alcohol abuse immunosuppression, and pulmonary infection. Curr Drug Abuse Rev 1: 56-67.

8. Wiley TE, McCarthy M, Breidi L, McCarthy M, Layden TJ (1998) Impact of alcohol on the histological and clinical progression of hepatitis $\mathrm{C}$ infection. Hepatology 28: 805-809

9. Prakash O, Mason A, Luftig RB, Bautista AP (2002) Hepatitis C virus (HCV) and human immunodeficiency virus type 1 (HIV-1) infections in alcoholics. Front Biosci 7: e286-300.

10. Baliunas D, Rehm J, Irving H, Shuper P (2010) Alcohol consumption and risk of incident human immunodeficiency virus infection: a meta-analysis. Int $\mathrm{J}$ Public Health 55: 159-166. 
Citation: Gavala A, Myrianthefs P, Venetsanou K, Baltopoulos G, Alevizopoulos G (2015) Alcohol Effects on TNF- $\alpha$ and IL-10 Production in an Ex-Vivo Model of Whole Blood Stimulated by LPS. J Psychiatry 18: 339 doi:10.4172/2378-5756.1000339

Page 5 of 5

11. Brown LA, Cook RT, Jerrells TR, Kolls JK, Nagy LE, et al. (2006) Acute and chronic alcohol abuse modulate immunity. Alcohol Clin Exp Res 30: 1624-1631.

12. Messingham KA, Faunce DE, Kovacs EJ (2002) Alcohol, injury, and cellular immunity. Alcohol 28: 137-149.

13. Kawakami M, Switzer BR, Herzog SR, Meyer AA (1991) Immune suppression after acute ethanol ingestion and thermal injury. J Surg Res 51: 210-215.

14. Bird MD, Choudhry MA, Molina PE, Kovacs EJ (2009) Alcohol and trauma: a summary of the Satellite Symposium at the 30th Annual Meeting of the Shock Society. Alcohol 43: 247-252.

15. Stoltz DA, Zhang P, Nelson S, Bohm RP Jr, Murphey-Corb M, et.al. (1999) Ethanol suppression of the functional state of polymorphonuclear leukocytes obtained from uninfected and simian immunodeficiency virus infected rhesus macaques. Alcohol Clin Exp Res 23: 878-884.

16. Mørland H, Johnsen J, Bjørneboe A, Bjørneboe GE, Drevon CA, et al. (1988) Reduced IgG Fc-receptor-mediated phagocytosis in human monocytes isolated from alcoholics. Alcohol Clin Exp Res 12: 755-759.

17. Szabo G, Catalano D, White B, Mandrekar P (2004) Acute alcohol consumption inhibits accessory cell function of monocytes and dendritic cells. Alcohol Clin Exp Res 28: 824-828.

18. Cook RT, Li F, Vandersteen D, Ballas ZK, Cook BL, et al. (1997) Ethano and natural killer cells. I. Activity and immunophenotype in alcoholic humans. Alcohol Clin Exp Res 21:974-980.

19. Szabo G, Mandrekar P, Dolganiuc A, Catalano D, Kodys K (2001) Reduced alloreactive T-cell activation after alcohol intake is due to impaired monocyte accessory cell function and correlates with elevated IL-10, IL-13, and decreased IFN gamma levels. Alcohol Clin Exp Res 25: 1766-1772.

20. Szabo G (1999) Consequences of alcohol consumption on host defence. Alcohol Alcohol 34: 830-841.

21. Myrianthefs $P$, Karatzas S, Venetsanou K, Grouzi E, Evagelopoulou P, et al. (2003) Seasonal variation in whole blood cytokine production after LPS stimulation in normal individuals. Cytokine 24: 286-292.

22. Larrick, JW, Hirata M, Zheng H, Zhong J, Bolin D, et.al. (1994)Nove granulocyte-derived peptide with lipopolysaccharide-neutralizing activity. J Immunol 152: 231-240.

23. Venetsanou K, Kaldis V, Kouzanidis N, Papazacharias Ch, Paraskevopoulos $\mathrm{J}$, et al. (2012) Measurement of tumour necrosis factor receptors for immune response in colon cancer patients. Clin Exp Med 12: 225-231.

24. Song K, Zhao XJ, Marrero L, Oliver P, Nelson S, et al. (2005) Alcohol reversibly disrupts TNF-alpha/TACE interactions in the cell membrane. Respir Res 6: 123.

25. Pang M, Bala S, Kodys K, Catalano D, Szabo G (2011) Inhibition of TLR8- and TLR4-induced Type I IFN induction by alcohol is different from its effects on inflammatory cytokine production in monocytes. BMC Immunol 12: 55

26. von Maltzan K, Tan W, Pruett SB (2012) Investigation of the role of TNF-ît converting enzyme (TACE) in the inhibition of cell surface and soluble TNF-Ît production by acute ethanol exposure. PLoS One 7: e29890.

27. Nelson S, Bagby GJ, Bainton BG, Summer WR (1989) The effects of acute and chronic alcoholism on tumor necrosis factor and the inflammatory response. $J$ Infect Dis 160: 422-429.

28. Goral J, Kovacs EJ (2005) In vivo ethanol exposure down-regulates TLR2 , TLR4-, and TLR9-mediated macrophage inflammatory response by limiting p38 and ERK1/2 activation. J Immunol 174: 456-463.

29. Pruett SB, Fan R, Zheng Q (2003) Acute ethanol administration profoundly alters poly $\mathrm{I}: \mathrm{C}$-induced cytokine expression in mice by a mechanism that is not dependent on corticosterone. Life Sci; 72:1825-1839.

30. Verma BK, Fogarasi M, Szabo G (1993) Down-regulation of tumor necrosis factor alpha activity by acute ethanol treatment in human peripheral blood monocytes. J Clin Immunol 13: 8-22.

31. Szabo G, Mandrekar P, Catalano D (1995) Inhibition of superantigen-induced $\mathrm{T}$ cell proliferation and monocyte IL- 1 beta, TNF-alpha, and IL-6 production by acute ethanol treatment. J Leukoc Biol 58: 342-350.

32. Szabo G, Mandrekar P, Girouard L, Catalano D (1996) Regulation of human monocyte functions by acute ethanol treatment: decreased tumor necrosis factor- alpha, interleukin-1 beta and elevated interleukin-10, and transforming growth factor- beta production. Alcohol Clin Exp Res 20: 900-907.
33. Szabo G (1998) Monocytes, alcohol use, and altered immunity. Alcohol Clin Exp Res 22: 216S-219S

34. Nair MP, Schwartz SA, Kronfol ZA, Hill EM, Sweet AM, et al. (1994) Suppression of tumor necrosis factor production by alcohol in lipopolysaccharide-stimulated culture. Alcohol Clin Exp Res 18: 602-607.

35. Mandrekar P, Bala S, Catalano D, Kodys K, Szabo G (2009) The opposite effects of acute and chronic alcohol on lipopolysaccharide-induced inflammation are linked to IRAK-M in human monocytes. J Immunol 183: 1320-1327.

36. Mandrekar P, Catalano D, Girouard L, Szabo G (1996) Human monocyte IL-10 production is increased by acute ethanol treatment. Cytokine 8: 567-577.

37. Szabo G, Mandrekar P, Dolganiuc A, Catalano D, Kodys K (2001) Reduced alloreactive T-cell activation after alcohol intake is due to impaired monocyte accessory cell function and correlates with elevated IL-10, IL-13, and decreased IFNgamma levels. Alcohol Clin Exp Res 25: 1766-1772.

38. Mandrekar P, Catalano D, White B, Szabo G (2006) Moderate alcohol intake in humans attenuates monocyte inflammatory responses: inhibition of nuclear regulatory factor kappa B and induction of interleukin 10. Alcohol Clin Exp Res 30: $135-139$

39. Norkina O, Dolganiuc A, Shapiro T, Kodys K, Mandrekar P, et al. (2007) Acute alcohol activates STAT3, AP-1, and Sp-1 transcription factors via the family of Src kinases to promote IL-10 production in human monocytes. J Leukoc Biol 82: $752-762$.

40. Szabo G, Mandrekar P, Oak S, Mayerle J (2007) Effect of ethanol on inflammatory responses. Implications for pancreatitis. Pancreatology 7: 115-123.

41. Kim DJ, Kim W, Yoon SJ, Choi BM, Kim JS, et al. (2003) Effects of alcoho hangover on cytokine production in healthy subjects. Alcohol 31: 167-170.

42. de Waal Malefyt R, Yssel H, de Vries JE (1993) Direct effects of IL-10 on subsets of human CD4+ T cell clones and resting T cells. Specific inhibition of IL-2 production and proliferation. J Immunol 150: 4754-4765.

43. Szabo G1 (1998) Monocytes, alcohol use, and altered immunity. Alcohol Clin Exp Res 22: 216S-219S.

44. Dolganiuc A, Szabo G (2009) In vitro and in vivo models of acute alcoho exposure. World J Gastroenterol 15: 1168-1177.

45. Pruett SB, Fan R, Zheng Q, Schwab C (2005) Differences in IL-10 and IL-12 production patterns and differences in the effects of acute ethanol treatment on macrophages in vivo and in vitro. Alcohol 37: 1-8.

46. Glover M, Cheng B, Deng X, Pruett S (2011) The role of glucocorticoids in the immediate vs. delayed effects of acute ethanol exposure on cytokine production in a binge drinking model. Int Immunopharmacol 11: 755-761.

47. Yaqoob P, Newsholme EA, Calder PC (1999) Comparison of cytokine production in cultures of whole human blood and purified mononuclear cells. Cytokine 11: 600-605.

48. Jones AW, Holmgren A (2009) Age and gender differences in blood-alcoho concentration in apprehended drivers in relation to the amounts of alcoho consumed. Forensic Sci Int 188: 40-45. 